Pacific Journal of Mathematics

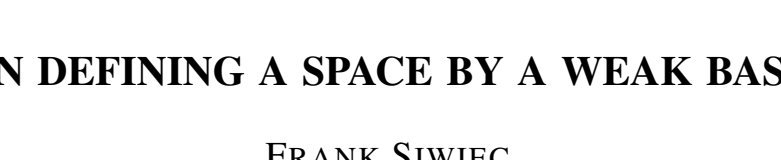




\title{
ON DEFINING A SPACE BY A WEAK BASE
}

\author{
FranK SiwieC
}

\begin{abstract}
Arhangel'skii has defined the concepts of a weak base and a $g$-first countable space $(=g f$-axiom of countability $=$ weak first axiom of countability). Here a $g$-second countable space and a $g$-metrizable space are defined and discussed, in particular in relation to metrizability.
\end{abstract}

We wish to discuss a means of defining topological spaces which may deserve to be better known. We begin with a slight modification of a definition due to Arhangel'skii [4, p. 129].

Definition 1.1. For a topological space $X$ and a point $x$ of $X$, a collection $T_{x}$ of subsets of $X$ is called a collection of weak neighborhoods of $x$ if each member of $T_{x}$ contains $x$, for any two members of $T_{x}$ their intersection is also a member of $T_{x}$, and the following is true: Letting $\mathscr{B}=\bigcup\left\{T_{x} \mid x \in X\right\}, \mathscr{B}$ is a weak base for $X$ if a set $U$ is open in $X$ if and only if for every point $x$ in $U$ there exists a $B \in T_{x}$ such that $B \subset U$.

1.2. It is clear that any set $X$ may be topologized by this natural method. To illustrate, we consider three known examples of spaces in which the topology is defined in this manner. Let $X$ be the plane, and for each point $x$ of $X$, we now define the collection $T_{x}$ for each of these three examples:

(a) $T_{x}$ consists of all sets which form a "plus at $x$ ", namely, sets which are the union of a horizontal open interval and a vertical open interval each of which contains the point $x$. (This example of J. Novak [27] may have its topology defined in some interesting alternative manners. See [1, Example 2], [3, p. 30], and [22].)

(b) $T_{x}$ consists of all sets "radial at $x$ ", namely, sets which contain a line segment through $x$ in every direction. (This is an example of Williams in [13].)

(c) $T_{x}=\left\{V_{n}(x) \mid n=1,2, \cdots\right\}$, where $V_{n}(x)$ is the translation of $V_{n}(0)$ by $x$, and $V_{n}(0)$ is that subset of the open $1 / n$ disc with center at the origin which is obtained by removing all points of the open second and fourth quadrants. (This is an example of Meyer in [21, 3.10].)

Example (c) is immediately seen to be a $g$-first countable space in the sense of the following definition also due to Arhangel'skii [4, p. 129]. The reader may also notice that example (a) is a $g$-first countable space, while example (b) is not. 
Definition 1.3. A space $X$ is $g$-first countable (this is our terminology; Arhangel'skii's is the weak first axiom of countability and the $g f$-axiom of countability) if its topology can be given by a weak base $\mathscr{B}=\bigcup\left\{T_{x} \mid x \in X\right\}$ where each $T_{x}$ is a countable collection.

1.4. A first countable space is indeed a $g$-first countable space. We now show that every $g$-first countable space is a sequential space in the sense of Franklin [11]. By definition, a space $X$ is sequential if every sequentially open set is open, where a set $U$ is said to be sequentially open if every sequence converging to a point in $U$ is eventually in $U$. Now let $X$ be a $g$-first countable space and let $U$ be a sequentially open set in $X$. Suppose $U$ is a nonopen set. Then there exists a point $x$ in $U$ such that no member of $T_{x}$ is contained in $U$. We may assume that $T_{x}=\left\{B_{n} \mid n=1,2, \cdots\right\}$ is a decreasing sequence of sets and for each positive integer $i$ we may choose a point $x_{i}$ in $B_{i}-U$. Then the sequence $\left\{x_{2}\right\}$ converges to $x$. For if $G$ is a neighborhood of $x$, then there exists a $B_{i} \in T_{x}$ such that $B_{i} \subset G$. So $x_{j} \in B_{i} \subset G$ for all $j \geqq i$. We then have the contradiction that $\left\{x_{i}\right\}$ converges to a point in $U$ and the sequence is not eventually in $U$.

Now we define two related concepts.

Definition 1.5. A space $X$ is $g$-second countable if for each positive integer $n$ there exists a subset $B_{n}$ of $X$ and corresponding to this subset there also exists a collection $\mathscr{B}_{n}$ of (not necessarily all) pairs $\left(B_{n}, x\right)$ with $x$ being a point of $B_{n}$, such that a set $U$ is open in $X$ if and only if for each point $x$ in $U$ there exists a pair $\left(B_{n}, x\right)$ for which $B_{n}$ is a subset of $U$. A regular space $X$ is $g$-metrizable if for each positive integer $n$ there exists a collection $\mathscr{B}_{n}$ of pairs $(B, x)$ where $B$ is a subset of $X$ and $x$ is a point of $B$, such that (i) each collection $\left\{B \mid(B, x) \in \mathscr{B}_{n}\right\}$ is locally finite, and (ii) a set $U$ is open in $X$ if and only if for each point $x$ in $U$, there exists a pair $(B, x)$ in $\bigcup\left\{\mathscr{B}_{n} \mid n=1,2, \cdots\right\}$ for which $B \subset U$.

Remarks 1.6. (a) Of course if one wishes to define a $g$-second countable topology on a set $X$, two additional properties must be satisfied: (i) For each point $x$ of $X$, there must be an $n$ such that $\left(B_{n}, x\right) \in \mathscr{B}_{n}$, and (ii) if $x \in X$ and $\left(B_{n_{1}}, x\right) \in \mathscr{B}_{n_{1}}$ and $\left(B_{n_{2}}, x\right) \in \mathscr{B}_{n_{2}}$, then there exists $n_{3}$ such that $\left(B_{n_{3}}, x\right) \in \mathscr{B}_{n_{3}}$ and $B_{n_{3}} \subset B_{n_{1}} \cap B_{n_{2}}$. Below, we will see more ways of forming $g$-second countable and $g$-metrizable spaces.

(b) It may also be of interest to point out that for a regular space, in particular for a $g$-metrizable space, the weak neighborhoods 
of a point may be chosen to be closed sets.

(c) Example 2.8 which will follow, shows that the words "locally finite" may not be replaced by the words "closure preserving" in the definition of a $g$-metrizable space without altering the concept. We do not know whether a $g$-metrizable space may be equivalently defined by using the word "discrete" in place of "locally finite".

1.7. Next we discuss some relations among the concepts. The Hausdorff axiom will be assumed in all that follows. Recall that a space $X$ is symmetrizable (respectively, semi-metrizable) if there exists a real-valued "distance" function $d$ for $X$ such that (i) for each $x$ and $y$ in $X, d(x, y)=d(y, x) \geqq 0$ and $d(x, y)=0$ if and only if $x=y$, and (ii) a set $F$ is closed in $X$ if and only if $d(x, F)>0$ for every $x$ in $X-F$ (respectively, (ii) $x \in \operatorname{cl} F$ if and only if $d(x, F)=0$.) We may note that condition (ii) for a symmetrizable space may be restated as follows: For every point $x$ of an open set $U$ there exists a ball centered at $x$ which is contained in $U$, and for every nonempty nonclosed set $A$ there exists a point $x$ in $X-A$ for which $d(x, A)=0$. (It is known that in a symmetrizable space a ball centered at $x$ need not be a neighborhood of $x$. For a semi-metrizable space a ball centered at $x$ is a neighborhood of $x$, but need not be open.) With this restatement, one can see that a symmetrizable space is $g$-first countable.

1.8. Next we show that a $g$-metrizable space is symmetrizable. First, it is clear that a $g$-metrizable space is $g$-first countable. Second it is also clear that a $g$-metrizable space is a $\sigma$-space (namely, a space with a $\sigma$-locally finite network, where a network is a collection $\mathscr{B}$ of sets such that whenever a point $x$ is in an open set $G$ there exists a $B \in \mathscr{B}$ such that $x \in B \subset G)$. By Siwiec and Nagata [31], a regular

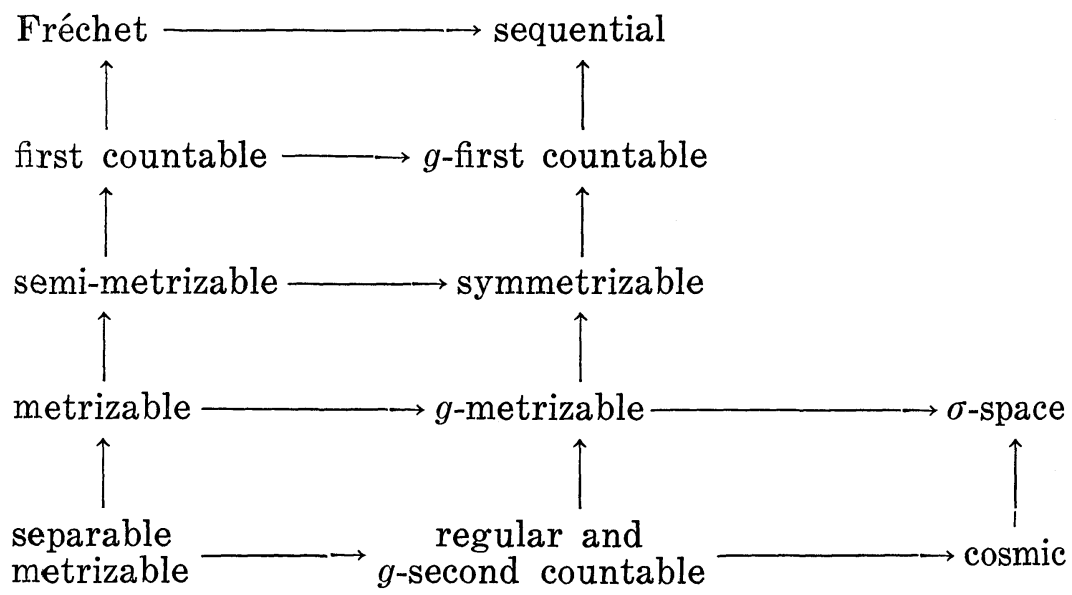


$\sigma$-space has a $\sigma$-discrete network. Then by Arhangel'skii [4, p. 130, Theorem 2.8], a $g$-first countable space with a $\sigma$-discrete network is symmetrizable. (However, we do not know whether a $g$-metrizable space satisfies the weak condition of Cauchy. See Proposition 1.18 and Problem 1.19.) We may also note that a regular $g$-second countable space is a cosmic space (namely, a regular space with a countable network). A space is Fréchet [11] if every accumulation point of a set is the limit of a sequence in the set. As a result, we have the relations shown in the diagram above.

Proposition 1.9. Each of the classes of spaces listed in the second column above is closed-hereditary (namely, a closed subspace of a space having the property also has the property).

THEOREM 1.10. If a space $X$ satisfies

(a) one of the properties in the second column and is also Fréchet, or

(b) one of the properties in the second column hereditarily, then $X$ satisfies the corresponding property in the first column.

Proof. We prove that a $g$-first countable Fréchet space is first countable. This result is stated by Arhangel'skii [4, p. 129] without proof. Let $B$ be a weak neighborhood of a point $x$ of a $g$-first countable Fréchet space $X$. We will show that $B$ is a neighborhood of $x$. Suppose it is not. Then by the Fréchet assumption, there is a sequence $\left\{x_{n}\right\}$ in $X-B$ which converges to $x$. Then the set $U=$ $X-\left\{x, x_{1}, x_{2}, \cdots\right\}$ is open, so that for each $p$ in $U$, there exists a $B_{p}$ in $T_{p}$ which is contained in $U$. Letting $U^{\prime}=U \cup\{x\}$, for each $p$ in $U^{\prime}$ there is then a $B_{p} \in T_{p}$ such that $B_{p} \subset U^{\prime}$, since if $p=x$ then $B_{p}=B \subset U^{\prime}$. By the $g$-first countable assumption, $U^{\prime}$ is open in $X$. But $x$ is in $U^{\prime}$, so that we have the contradiction that $\left\{x_{n}\right\}$ does not converge to $x$. Thus $x$ is in the interior of $B$.

It is easily seen, as stated by Arhangel'skii in [4, p. 131] that a symmetrizable space is semi-metrizable if it is first countable (or Fréchet).

Finally, if assumption (b) is made, then the space $X$ is hereditarily a sequential space, thus hereditarily a $k$-space, and thusFréchet by Arhangel'skii [5], so that assumption (a) holds. Our proofs are now complete.

Since the definition of a $g$-second countable space and especially that of a $g$-metrizable space are complicated, it may be difficult in a particular instance to determine whether a space satisfies these conditions. Theorem 1.10 has already presented two means of determining this, and we now present some additional and more useful means. 
Observation 1.11. A countable space which is $g$-first countable is $g$-second countable. Thus, a countable regular space which is $g$-first countable is $g$-metrizable.

For the following results we will need several concepts which generalize first countability (see [29] for a survey of these concepts). An accessibility space has been characterized as a space $X$ for which every quotient mapping onto $X$ is a pseudo-open (equivalently, hereditarily quotient) mapping. A space $X$ is said to be accumulation complete if whenever a point $x$ is a cluster point of a sequence in $X$, there is a subsequence which converges to $x$, or equivalently, every countable subspace of $X$ is Fréchet. A space $X$ has countable tightness provided that whenever a point $x$ is an accumulation point of a set $F$ there exists a countable set $C$ in $F$ such that $x$ is an accumulation point of $C$. A space $X$ is a $k^{\prime}$-space if whenever a point $x$ is an accumulation point of a set $A$ in $X$, there exists a compact set $K$ such that $x$ is an accumulation point of $A \cap K$. A space $X$ is a $q$-space if for every point $x$ of $X$ there exists a sequence $\left\{U_{n}(x) \mid n \in N\right\}$ of neighborhoods of $x$ such that if $x_{n}$ is in $U_{n}$ for each $n$, then the sequence $\left\{x_{n}\right\}$ has a cluster point $x^{\prime}$. A space $X$ is singly $b i$-quasi-k if whenever a point $x$ is an accumulation point of a set $F$ there exists a $q$-sequence $\left\{A_{n} \mid n \in N\right\}$ in $X$ such that $x \in \operatorname{cl}\left(F \cap A_{n}\right)$ for all $n$. Here a $q$-sequence $\left\{A_{n} \mid n \in N\right\}$ of sets is defined as a decreasing sequence of sets for which there exists a countably compact set $A$ contained in all $A_{n}$ and such that every neighborhood of $A$ contains some $A_{n}$.

Lemma 1.12. If a space $X$ has countable tightness and every countable subspace is singly bi-quasi-k, then $X$ itself is singly biquasi-k.

Proof. Let $x$ be an accumulation point of a set $F$ in $X$. Then there exists a countable set $C$ in $F$ such that $x$ is an accumulation point of $C$. Let $D=C \cup\{x\}$. Then $D$ is singly bi-quasi-k. Thus there exists a $q$-sequence $\left\{A_{n} \mid n \in N\right\}$ in $D$ such that $x$ is a $D$-accumulation point of $C \cap A_{n}$ for every $n$. Thus $\left\{A_{n} \mid n \in N\right\}$ is a $q$-sequence in $X$, and $x$ is an accumulation point of $C \cap A_{n}$ in $X$ for every $n$.

Theorem 1.13. A g-metrizable space $X$ is metrizable if $X$ is also:

( a Fréchet,

(b) accumulation complete,

(c) every countable subspace of $X$ is Fréchet,

(d) every countable subspace of $X$ is a $k^{\prime}$-space, 
(e) every countable subspace of $X$ is singly bi-quasi-k,

(f) q-space,

(g) accessibility space.

Proof. Consider case (g) first. If $X$ is a $g$-metrizable space then $X$ is sequential and thus as is well-known, a quotient image of a metric space under a mapping $f$. Assuming $X$ to be an accessibility space, the mapping $f$ is then pseudo-open, so that $X$ is a Fréchet space (being: a pseudo-open image of a metric space). Thus we have case (a), which was proved in Theorem 1.10. All other cases of this theorem imply case (e) as may be seen by the reader by checking this directly or by recalling known results (see [29]). Thus we now prove case (e). Notice that a $g$-metrizable space has countable tightness since this concept is weaker than the concept of a sequential space. By Lemma 1.12, $X$ is then singly bi-quasi- $k$. But a regular $\sigma$-space, in particular a $g$-metrizable space, is known to have the property that every point is a countable intersection of closed neighborhoods. The space $X$ having this property and being single biquasi-k, is then necessarily a Fréchet space, so that case (a) applies again.

REMARK 1.14. Clearly Theorem 1.13 holds in a similar version for other concepts in the second column of the diagram given above. In particular, a regular $g$-second countable space is second countable if the space satisfies any of the seven conditions of Theorem 1.13. With some additional assumptions, a like result holds for symmetrizability.

We may characterize a $g$-metrizable space by using some concepts of Guthrie, Michael, and O'Meara related to networks. Let us define these. A k-network $\mathscr{P}$ for a space $X$ is a family of subsets of $X$ such that if $U$ is an open neighborhood of a compact set $C$, then there is a finite union $R$ of members of $\mathscr{P}$ such that $C \subset R \subset U$. A collection $\mathscr{P}$ of subsets of $X$ is a cs-network for $X$ if whenever $U$ is an open set which contains a convergent sequence $\left\{z_{n}\right\}$ and also contains the limit $z$ of the convergent sequence $\left\{z_{n}\right\}$, then $\left\{z, z_{n}\right.$, $\left.z_{n+1}, \cdots\right\} \subset P \subset U$ for some $n$ and for some $P$ in $\mathscr{P}$. A space $X$ is an $\aleph$-space if it is regular and has a $\sigma$-locally finite $k$-network [28]. A space $X$ is an $\boldsymbol{\aleph}_{0}$-space if it is regular and has a countable $k$-network [23]. A space $X$ is a $c s-\sigma$-space if it is regular and has a $\sigma$-locally finite $c s$-network [16].

The writer is grateful to Dennis Burke for some considerably useful remarks in the following. 
THEOREM 1.15. The following are equivalent for a space $X$ :

(a) $X$ is a g-metrizable space,

(b) $X$ is g-first countable and a cs-o-space.

The following are equivalent for a regular space $X$ : (D. Burke and J. Guthrie [15]),

(a) $X$ is g-second countable,

(b) $X$ is g-first countable and an $\boldsymbol{\aleph}_{0}$-space,

(c) $X$ is g-first countable and has a countable cs-network.

Proof. We will prove the equivalence of (a) and (b) of the first statement. The equivalence of the latter three conditions may be proved in a like manner. We already known that a $g$-metrizable space is $g$-first countable. We will show that a $g$-metrizable space is a $c s-\sigma$-space. Let $\left\{z_{n}\right\}$ be a sequence converging to a point $z$ in the $g$-metrizable space $X$. Let $G$ be an open set containing both the convergent sequence $\left\{z_{n}\right\}$ and also the limit $z$. Since $Z=\left\{z, z_{1}, z_{2} \cdots\right\}$ is a compact metrizable space, Theorem 1.10 shows that every weak neighborhood of a point in $Z$ is then a neighborhood (in $Z$ ) of the point. Let $B$ be a weak neighborhood of the limit point $z$, such that $B \subset G$. Then $z \in \operatorname{Int}_{z} B$. Thus there exists an $n$ such that $\left\{z_{n}, z_{n+1}\right.$, $\cdots\} \subset B$. Now let $X$ be a space which is $g$-first countable and a $c s-\sigma$-space. Let $\bigcup\left\{\mathscr{P}_{n} \mid n \in N\right\}$ be the $\sigma$-locally finite $c s$-network for $X$, and suppose that $\mathscr{P}_{n} \subset \mathscr{P}_{n+1}$ for every $n$. Let $\left\{S_{n}(x) \mid n \in N\right\}$ be the weak neighborhood base at a point $x$ in $X$. Let

$$
\mathscr{B}_{n}=\left\{(B, x) \mid S_{n}(x) \subset B \in \mathscr{P}_{n}\right\} \text {. }
$$

We wish to show that the collections $\mathscr{B}_{n}$ are collections of pairs as in the definition of a $g$-metrizable space. Let $U$ be a set such that for each point $x$ in $U$ there exists a pair $(B, x) \in \bigcup\left\{\mathscr{B}_{n} \mid n \in N\right\}$ such that $B \subset U$. Then for each $x$ in $U$, there exists an $n$ such that $S_{n}(x) \subset U$. By the definition of a $g$-first countable space, $U$ is then an open set. Conversely, let $U$ be an open neighborhood of a point $x$ in $X$. We wish to show that there exists an integer $n$ and a pair $(B, x) \in \mathscr{B}_{n}$ such that $B \subset U$. If not, then for all $n, S_{n}(x) \not \subset B$ for all $B$ in $\mathscr{P}_{n}$ such that $x \in B \subset U$. Thus for each $n$ and for each $B \in \mathscr{P}_{n}$ such that $x \in B \subset U$, there exists a point $x(n, B) \in S_{n}(x)-B$. The collection of such $x(n, B)$ is countable and upon being enumerated converges to $x$. Since $x$ is in $U$, there exists an integer $j$ such that $x(l, B)$ is in $U$ for all $x(l, B)$ chosen above with $l \geqq j$. Because $X$ is a $c s$ - $\sigma$-space, there exists an integer $k \geqq j$ such that all $x(l, B)$ chosen above are in some $B^{\prime}$ of $\mathscr{P}_{k}$ for $l \geqq k$, and $x$ is also in $B^{\prime}$. We then have the contradiction that $x\left(k, B^{\prime}\right)$ is in $B^{\prime}$. Thus there 
exists a pair $(B, x)$ as desired, and $X$ is $g$-metrizable.

Problems 1.16. (a) Is every $g$-metrizable space, a normal space? (b) Is every normal, $g$-metrizable space also paracompact? (c) Is every separable, $g$-metrizable space, a $g$-second countable space? (d) May a $g$-metrizable space be characterized by means of a distance function? It might be noted that one natural way of attempting to give such a characterization actually yields a metrizable space. Specifically, it may be easily seen that if $X$ is a set with a metric $d$ defined on $X \times X$, then each of the following define a topology on $\mathrm{X}$ and the topologies so defined are equivalent: (i) The collection of all $1 / n$ balls forms a base. (ii) A point $x$ is in the closure of a set $F$ if and only if $d(x, F)=0$. (iii) A set $F$ is closed if and only if $d(x, F)>0$ for every $x$ in $X-F$. (e) Arhangel'skii in [4, p. 133] has referred to a symmetric of a symmetrizable space as satisfying condition $(K)$ if the distance between any two disjoint compact sets is positive. We have not been able to determine whether the following conditions for a regular space $X$ are equivalent: (i) $X$ is a $g$-metrizable space, (ii) $X$ is $g$-first countable and a $c s-\sigma$-space, (iii) $X$ is $g$-first countable and an $\aleph$-space, (iv) $X$ is symmetrizable by a symmetric which satisfies condition $(K)$. Notice that Guthrie in [16] has shown that every $c s-\sigma$-spaces is an $X$-space.

Regarding mappings, all of which are assumed to be continuous, we also have some results. A mapping $f: X \rightarrow Y$ is a $\pi$-mapping if for each $y$ in $Y$ and each open neighborhood $U$ of $y$, the distance of $f^{-1}(y)$ from $X-f^{-1}(U)$ is positive. A mapping $f: X \rightarrow Y$ is compact if $f^{-1}(y)$ is compact for each $y \in Y$.

Proposition 1.17.

(a) (Arhangel'skii [2]) A quotient $\pi$-image of a metric space is symmetrizable. (See some related results given below.)

(b) A quotient compact image of a second countable space is $g$-second countable.

(c) (D. Burke) A quotient $\pi$-image of a separable metric space is $g$-second countable.

Proof. Proof of (b): Let $f$ be a quotient mapping of a second countable space $X$ onto $Y$. Let $\mathscr{B}$ be a countable base for $X$ which is closed under finite unions (that is, all finite unions of members of $\mathscr{B}$ are also members of $\mathscr{B})$. For each $B_{n} \in \mathscr{B}$, let

$$
\mathscr{B}_{n}=\left\{\left(f\left(B_{n}\right), y\right) \mid f^{-1}(y) \subset B_{n}\right\} .
$$

Then it is easily seen that this is the desired collection in $Y$. Proof 
of (c): The image is symmetrizable by (a) and an $\aleph_{0}$-space by Michael [23, Prop. 2.1], thus $g$-second countable by Theorem 1.15.

Proposition 1.18. (Arhangel'skii, Burke and Stoltenberg, Coban, Kofner). The following are equivalent:

(a) $Y$ is symmetrizable with a symmetric satisfying the weak condition of Cauchy (namely, every convergent sequence has a Cauchy subsequence, equivalently, for each nonclosed set $A$ and for each $\varepsilon>0$ there exist points $x$ and $y$ in $A$ such that $d(x, y)<\varepsilon)^{1}$.

(b) $Y$ has a semi-refinement (namely, a sequence $\left\{\mathscr{V}_{n}\right\}$ of (not necessarily open) covers satisfying the condition that for each point $y$ in $Y$ and each neighborhood $U$ of $y$ there exists an $n$ such that $\left.\operatorname{St}\left(y, \mathscr{C}_{n}\right) \subset U\right)^{2}$ satisfying the weak condition of Cauchy ${ }^{1}$.

(c) $Y$ is a quotient $\pi$-image of a metric space.

Credits. Arhangel'skii in [2] showed that (c) implies (a). Kofner stated the converse in [19, Theorem 11], with his proof being given in [20, Theorem 4]. Burke and Stoltenberg in [8, Theorem 3.2] proved the equivalence of a symmetrizable space and a space which has a semi-refinement ${ }^{3}$, thus proving the equivalence of (a) and (b). Coban in [10] claimed to have proved the equivalence of $\left(b^{\prime}\right)$ and (c), where $\left(b^{\prime}\right)$ is: $Y$ has a semi-refinement. Burke and Stoltenberg (in the above mentioned paper) noticed Coban's claim to be false and gave a counterexample of a symmetrizable space which does not satisfy (a). (The same example was also discussed by Kofner in [19].) However, if one modifies Coban's statement to include the weak condition of Cauchy, and if one modifies his proof accordingly, then his proof is correct as given.

Problem 1.19. Is every $g$-metrizable space a quotient $\pi$-image (or quotient compact image) of a metric space? Burke has pointed out that an open compact image of a metric space need not be $g$-metrizable. For let $Y$ be the space of Example B of Bing [6]. $Y$ is metacompact, developable, and not metrizable, so not $g$-metrizable. But $Y$ is an open compact image of a metric space, being a metacompact developable space.

1.20. Now let us consider another application of mappings.

1 The weak condition of Cauchy has been considered by writers in numerous different forms. Two formulations are given in statement (a), another, suitable for statement (b) is: if $D$ is a set for which there exists an $n$ such that $\operatorname{St}\left(x, \mathscr{V}_{n}\right) \cap D=\{x\}$ for all $x$ in $D$, then $D$ is closed. See Burke [7], Kofner [20, Proposition 3].

2 This is a slight modification of, but equivalent to, Coban's definition [10].

3 They also have another characterization of such a space (Theorem 3.1). 
Arhangel'skii in [4, p. 129] has stated the interesting result that the Morita and Hanai, and Stone theorem is true for a $g$-first countable range space, that is, a closed image of a metric space which is $g$-first countable is itself metrizable. Though this result may be proved more directly, we do so by showing that a $g$-first countable space has the following property: If $\left\{F_{n}\right\}$ is a decreasing sequence of closed sets, each of which has a point $x$ as a common accumulation point, then for each $n$, there exists a subset $A_{n}$ of $F_{n}$, closed in $X$, such that the union of all of the $A_{n}$ is not a closed set. We denote the weak base at the point $x$ by $T_{x}=\left\{B_{n}(x)\right\}$, and we fix the index $n$. Since $F_{n}-\{x\}$ is a nonclosed set, there exists a point $p$ in the complement of $F_{n}-\{x\}$ such that no member of $T_{p}$ is contained in $X-$ $\left(F_{n}-\{x\}\right)$. So for all $i$, there exists a point $x_{i}$ in $B_{i}(p) \cap\left(F_{n}-\{x\}\right)$. Then the sequence of $x_{i}$ converges to $p$. But since this sequence is contained in $F_{n}, p \in F_{n}$, and $p=x$. Thus for all $n$, there exists a point $x_{n}$ in $B_{n}(x) \cap\left(F_{n}-\{x\}\right)$. The sequence $\left\{x_{n}\right\}$ converges to $x$ and $x_{n} \neq x$ for all $n$. For each $n$, let $A_{n}$ be the singleton $\left\{x_{n}\right\}$. Then each $A_{n}$ is a closed set contained in $F_{n}$, but $\bigcup\left\{A_{n} \mid n=1,2, \cdots\right\}=$ $\left\{x_{1}, x_{2}, \cdots\right\}$ is not closed.

The property stated above is a slight weakening of a property of Michael in [25], in particular, it is property 9.1(b) stated for closed sets $F_{n}$. Michael uses this property to prove a generalization of the Morita and Hanai, and Stone theorem, but in actuality, this property for closed sets suffices in Michael's proof. Thus by his Theorem 9.9 and Corollary 9.10, we have the desired result stated below.

Proposition 1.21. Let $Y$ be a closed image of a metric space. Then $Y$ is metrizable if $Y$ is g-first countable, or more generally, if the following property is satisfied in $Y$ : if $\left\{F_{n}\right\}$ is a decreasing sequence of closed subsets of $Y$ each of which has a point $y$ as a common accumulation point, then for each $n$, there exists a closed set $A_{n}$ contained in $F_{n}$ such that the union of all of the $A_{n}$ is not a closed set.

For a somewhat better result and more background on this topic, see [30] and a forthcoming paper of Michael, Olson, and the writer.

2. In this section we present some examples.

2.1. A space which is regular, countable (thus cosmic), and as is easily verified, $g$-second countable, but is not Fréchet, thus not metrizable: Example 5.1 of Franklin in [12].

2.2. A space which is second countable and thus satisfies the 
condition for a $g$-metrizable space except for not being regular: Any nonregular, second countable space.

2.3. A space which is regular and countable (thus cosmic), but is not sequential, thus not $g$-second countable: The example of Arens given in [18, p. $77 \$ 2 \mathrm{E}]$.

2.4. A cosmic, Fréchet space which is not first countable, thus not $g$-first countable: The space of rational numbers with the integers identified to a point and the quotient (or identification) topology.

2.5. A space which is compact and Fréchet, but not $g$-first countable, since it is not first countable: The one-point compactification of an uncountable discrete space.

2.6. A regular, hereditarily Lindelof, first countable space which is not symmetrizable: The half-open interval topology of Sorgenfrey on the real line.

2.7. A space which is regular, hereditarily Lindelof, and semimetrizable, but not a $\sigma$-space, thus not $g$-metrizable: An example of Michael (concerning normality of products) [24, the space $Y$ of Example 1.3].

2.8. A regular space which has a $\sigma$-closure preserving base (namely, an $M_{1}$-space in the terminology of Ceder [9]), which is also cosmic, and semi-metrizable, but not $g$-metrizable (since it is not metrizable). Though this space is not $g$-metrizable, it does satisfy the definition of $g$-metrizability if the words "locally finite" of the definition are replaced by the words "closure-preserving": An example of Nagata in [26]. (The example may also be found in Ceder's paper $[9,9.2]$.

2.9. A space which is completely regular, developable (thus semimetrizable), a $\sigma$-space, separable, and connected, but not $g$-metrizable (since it is not metrizable): The tangent disc space of Niemytzki (see for example [14, Problem 3K]).

2.10. A metrizable space which is not cosmic: A discrete space of uncountable cardinality.

2.11. A regular, $g$-metrizable space which is not metrizable and not $g$-second countable: The discrete union of Examples 2.1 and 2.10.

2.12. A regular, $M_{1}, g$-second countable space $X$ such that $X \times X$ 
is not $g$-second countable, in fact, not even a $k$-space: An example of Kofner [19, Example 3].

\section{REFERENCES}

1. B. A. Anderson, A class of topologies with $T_{1}$-complements, Fund. Math., 69 (1970), 267-277.

2. A. Arhangel'skii, Behavior of metrizability under factor mappings, Soviet Math. Dokl., 6 (1965), 1187-1190.

3. - Bicompact sets and the topology of spaces, Trans. Moscow Math. Soc., 13 (1965), 1-62.

4. - Mappings and spaces, Russian Math. Surveys, 21 (1966), 115-162.

5. — A characterization of very k-spaces, Czech. Math. J., 18 (1968), 392-395.

6. R. H. Bing, Metrization of topological spaces, Canad. J. Math., 3 (1951), 175-186.

7. D. K. Burke, Cauchy sequences in semimetric spaces, to appear.

8. D. K. Burke and R. A. Stoltenberg, Some properties of $\pi$-images of metric spaces, 1970.

9. J. G. Ceder, Some generalizations of metric spaces, Pacific J. Math., 11 (1961), 105125.

10. M. M. Coban, Mappings of metric spaces, Soviet Math. Dokl., 10 (1969), 258-260.

11. S. P. Franklin, Spaces in which sequences suffice, Fund. Math., 57 (1965), 107115.

12. - Spaces in which sequences suffice. II, Fund. Math., 61 (1967), 51-56.

13. S. P. Franklin and S. W. Williams, Nonregular separable spaces, Amer. Math. Monthly, 75 (1968), 208.

14. L. Gillman and M. Jerison, Rings of Continuous Functions, Van Nostrand, Princeton, 1960.

15. J. A. Guthrie, A characterization of $\boldsymbol{\aleph}_{0}$-spaces, Gen. Topology and Appl., 1 (1971), $105-110$.

16. - Mapping spaces and cs-networks, Pacific J. Math., 47 (1973), 465-471.

17. N. Howes and R. Chandler, Accumulation complete and sequential spaces, Not. Amer. Math. Soc., 16 (1969), 850.

18. J. L. Kelley, General Topology, Van Nostrand, Princeton, 1955.

19. J. A. Kofner, On a new class of spaces and some problems of symmetrizability theory, Soviet Math. Dokl., 10 (1969), 845-848.

20. - On two problems in the theory of symmetrizability, Bull. Acad. Polon., 18 (1970), 81-87, (in Russian).

21. P. R. Meyer, Topologies with the Stone-Weierstrass property, Trans. Amer. Math. Soc., 126 (1967), 236-243.

22. P. R. Meyer and R. Cougar, Topologies of separate continuity in a cross space, Amer. Math. Monthly, 75 (1968), 1128.

23. E. Michael, $\aleph_{0}$-spaces, J. Math. and Mech., 15 (1966), 983-1002.

24. - Paracompactness and the Lindelof property in finite and countable Cartesian products, Comp. Math., 23 (1971), 199-214.

25. - - A quintuple quotient quest, Gen. Topology and Appl., 2 (1972), 91-138.

26. J. Nagata, A contribution to the theory of metrization, J. Inst. Polytech., Osaka City Univ., 8 (1957), 185-192.

27. J. Novak, Induktion partiell stetiger Funktionen, Math. Ann., 118 (1942), 449461.

28. P. O'Meara, On paracompactness in function spaces with the compact-open topology, Proc. Amer. Math. Soc., 29 (1971), 183-189.

29. F. Siwiec, Generalizations of the first axiom of countability, to appear in the Rocky Mountain J. Math. 
30. F. Siwiec, On the Theorem of Morita and Hanai, and Stone, Second Pittsburgh International Topology Conf., 1972, Lecture Notes in Mathematics, no. 378, Springer Verlag, 1974, 449-454.

31. F. Siwiec and J. Nagata, A note on nets and metrization, Proc. Japan Acad., 44 (1968), 623-627.

Received November 8, 1972 and in revised form March 29, 1974.

John Jay College of the City University of New York 



\section{PACIFIC JOURNAL OF MATHEMATICS}

EDITORS

RICHARD ARENS (Managing Editor)

University of California

Los Angeles, California 90024

R. A. Beaumont

University of Washington

Seattle, Washington 98105

J. DugundJI

Department of Mathematics

University of Southern California

Los Angeles, California 90007

D. Gilbarg and J. Milgram

Stanford University

Stanford, California 94305

\section{ASSOCIATE EDITORS}

E. F. BECKENBACH

B. H. NeumanN

F. WOLF

K. YOSHIDA

\section{SUPPORTING INSTITUTIONS}

UNIVERSITY OF BRITISH COLUMBIA CALIFORNIA INSTITUTE OF TECHNOLOGY

UNIVERSITY OF CALIFORNIA

MONTANA STATE UNIVERSITY

UNIVERSITY OF NEVADA

NEW MEXICO STATE UNIVERSITY

OREGON STATE UNIVERSITY

UNIVERSITY OF OREGON

OSAKA UNIVERSITY

\author{
UNIVERSITY OF SOUTHERN CALIFORNIA \\ STANFORD UNIVERSITY \\ UNIVERSITY OF TOKYO \\ UNIVERSITY OF UTAH \\ WASHINGTON STATE UNIVERSITY \\ UNIVERSITY OF WASHINGTON \\ $\stackrel{*}{*} \stackrel{*}{*}{ }^{*}{ }^{*}{ }^{2}$ AMERICAN MATHEMATICAL SOCIETY \\ NAVAL WEAPONS CENTER
}




\section{Pacific Journal of Mathematics}

\section{Vol. 52, No. $1 \quad$ January, 1974}

David R. Adams, On the exceptional sets for spaces of potentials ............ 1

Philip Bacon, Axioms for the Čech cohomology of paracompacta ............ 7

Selwyn Ross Caradus, Perturbation theory for generalized Fredholm operators ..... 11

Kuang-Ho Chen, Phragmén-Lindelöf type theorems for a system of nonhomogeneous equations ............................ 17

Frederick Knowles Dashiell, Jr., Isomorphism problems for the Baire classes .......

M. G. Deshpande and V. K. Deshpande, Rings whose proper homomorphic images are right subdirectly irreducible . . . . . . . . . . . . . . . . . . . . . . . . .

Mary Rodriguez Embry, Self adjoint strictly cyclic operator algebras .............

Paul Erdős, On the distribution of numbers of the form $\sigma(n) / n$ and on some related

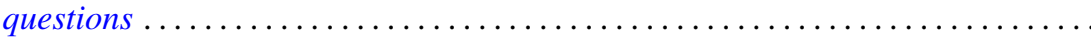

Richard Joseph Fleming and James E. Jamison, Hermitian and adjoint abelian

operators on certain Banach spaces ............................

Stanley P. Gudder and L. Haskins, The center of a poset .................. 85

Richard Howard Herman, Automorphism groups of operator algebras . . . ........

Worthen N. Hunsacker and Somashekhar Amrith Naimpally, Local compactness of families of continuous point-compact relations ....................

Donald Gordon James, On the normal subgroups of integral orthogonal groups ....

Eugene Carlyle Johnsen and Thomas Frederick Storer, Combinatorial structures in

loops. II. Commutative inverse property cyclic neofields of prime-power

order.

Ka-Sing Lau, Extreme operators on Choquet simplexes . . . . . . . . . . . . . . 129

Philip A. Leonard and Kenneth S. Williams, The septic character of 2, 3, 5 and $7 \ldots 143$

Dennis McGavran and Jingyal Pak, On the Nielsen number of a fiber map ........ 149

Stuart Edward Mills, Normed Köthe spaces as intermediate spaces of $L_{1}$ and

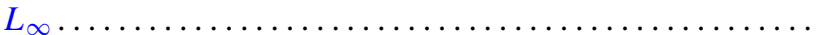

Philip Olin, Free products and elementary equivalence. .

Louis Jackson Ratliff, Jr., Locally quasi-unmixed Noetherian rings and ideals of the principal class.

Seiya Sasao, Homotopy types of spherical fibre spaces over spheres ...

Helga Schirmer, Fixed point sets of polyhedra ...

Kevin James Sharpe, Compatible topologies and continuous irreducible

representations.

Frank Siwiec, On defining a space by a weak base . . . . . . . . . . . . . . . 233

James McLean Sloss, Global reflection for a class of simple closed curves ....... 247

M. V. Subba Rao, On two congruences for primality . .

Raymond D. Terry, Oscillatory properties of a delay differential equation of even

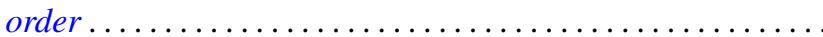

Joseph Dinneen Ward, Chebyshev centers in spaces of continuous functions . .

Robert Breckenridge Warfield, Jr., The uniqueness of elongations of Abelian

groups...

V. M. Warfield, Existence and adjoint theorems for linear stochastic differential

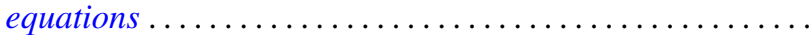

